\title{
Solution of Sequential Hadamard Fractional Differential Equations by Variation of Parameter Technique
}

\author{
Mohammed M. Matar \\ Mathematics Department, Al-Azhar University-Gaza, Gaza, State of Palestine \\ Correspondence should be addressed to Mohammed M. Matar; mohammed_mattar@hotmail.com
}

Received 30 September 2017; Accepted 4 January 2018; Published 26 February 2018

Academic Editor: Alexander Domoshnitsky

Copyright (C) 2018 Mohammed M. Matar. This is an open access article distributed under the Creative Commons Attribution License, which permits unrestricted use, distribution, and reproduction in any medium, provided the original work is properly cited.

We obtain in this article a solution of sequential differential equation involving the Hadamard fractional derivative and focusing the orders in the intervals $(1,2)$ and $(2,3)$. Firstly, we obtain the solution of the linear equations using variation of parameter technique, and next we investigate the existence theorems of the corresponding nonlinear types using some fixed-point theorems. Finally, some examples are given to explain the theorems.

\section{Introduction}

In recent years, there has been a great development in the study of fractional differential equations. This advancement is ranging from the theoretical analysis of the subject to analytical and numerical techniques (see [1-3] and the references cited therein). Among the theoretic approach, the existence theory of solutions for fractional differential models has gained attentions of many authors. Most of them have focused on using Riemann-Liouville and Caputo derivatives in representing the underlying fractional differential equation (see [4-10]). Another kind of fractional derivative is Hadamard type which was introduced in 1892 [11]. This derivative differs from aforementioned derivatives in the sense that the kernel of the integral in the definition of Hadamard derivative contains logarithmic function of arbitrary exponent. A detailed description of Hadamard fractional derivative and integral can be found in $[12,13]$. Recently, the existence and uniqueness of solution for fractional differential equations in Hadamard sense were introduced in many faces by several authors $([6,7]$ and references therein). We add in this article a new idea concerning the sequential definition of Hadamard fractional operator with constant coefficients of order less than three. For $q \in(2,3)$, the Hadamard fractional differential equation can be transformed to classical Euler-Cauchy second-order nonhomogeneous differential equation that can be solved by variation of parameter technique.

More precisely, we consider the nonlinear Hadamard fractional differential equations given by

$$
\begin{aligned}
& { }^{H} D_{a+}^{q} x(t)=f_{1}(t, x(t)), \quad q \in(0,1), \\
& x(a+)=0, \\
& \left({ }^{H} D_{a+}^{q}+\gamma^{H} D_{a+}^{q-1}\right) x(t)=f_{2}(t, x(t)), \quad q \in(1,2), \\
& x(a+)=x^{(1)}(a+)=0, \\
& \left({ }^{H} D_{a+}^{q}+\lambda_{1}{ }^{H} D_{a+}^{q-1}+\lambda_{2}{ }^{H} D_{a+}^{q-2}\right) x(t)=f_{3}(t, x(t)), \\
& x(a+)=x^{(1)}(a+)=x^{(2)}(a+)=0,
\end{aligned}
$$

where $t \in J=[a, b], 1 \leq a<b<+\infty$, and $f_{k}: J \times \mathbb{R} \rightarrow \mathbb{R}$ is a given continuous function for each $k=1,2,3$. Here, ${ }^{H} D_{a+}^{q}$ is the Hadamard fractional derivative, and $\gamma, \lambda_{1}$, and $\lambda_{2}$ are constants. 


\section{Linear Fractional Differential Equations}

We introduce some basic ideas of fractional calculus that may be used in the sequel of this article [12].

Definition 1. The Hadamard fractional integral of order $q>0$ is defined as

$$
\mathscr{f}_{a+}^{q} h(t)=\frac{1}{\Gamma(q)} \int_{a}^{t}\left(\ln \frac{t}{s}\right)^{q-1} \frac{h(s)}{s} d s
$$

provided that the integral exists.

Definition 2. The Hadamard derivative of fractional order $q>$ 0 is defined as

$$
\begin{aligned}
{ }^{H} D_{a+}^{q} h(t)=\delta^{n}\left(\mathscr{J}_{a+}^{q} h\right)(t), \\
\\
\quad n-1<q<n, n=[q]+1, \delta=t \frac{d}{d t},
\end{aligned}
$$

where $[q]$ denotes the integer part of the real number $q$.

Let $C(J, \mathbb{R})$ denote the Banach space of all real valued continuous functions defined on $J$, and $C_{\delta}^{n}(J, \mathbb{R})$ denotes the Banach space of all real valued functions $f$ such that $\delta^{n} f \in$ $C(J, \mathbb{R})$.

Lemma 3 (see $[12])$. Let $x \in C_{\delta}^{n}(J, \mathbb{R}), h \in C(J, \mathbb{R})$, and $c_{k} \in$ $\mathbb{R}$; then,

$$
\begin{aligned}
\mathscr{J}_{a+}^{q} \mathscr{J}_{a+}^{p} h(t) & =\mathscr{J}_{a+}^{q+p} h(t), \\
{ }^{H} D_{a+}^{q}\left(\mathscr{J}_{a+}^{q} x(t)\right) & =x(t), \\
\mathscr{J}_{a+}^{q}\left({ }^{H} D_{a+}^{q} x(t)\right)= & x(t)+c_{1}\left(\ln \frac{t}{a}\right)^{q-1} \\
& +c_{2}\left(\ln \frac{t}{a}\right)^{q-2}+\cdots \\
& +c_{n}\left(\ln \frac{t}{a}\right)^{q-n} .
\end{aligned}
$$

The following result is obvious by Lemma 3.

Lemma 4. Let $x \in C_{\delta}^{1}(J, \mathbb{R})$, and $g_{1} \in C(J, \mathbb{R})$. The fractional differential equation

$$
\begin{aligned}
{ }^{H} D_{a+}^{q} x(t) & =g_{1}(t), \quad t \in J, 0<q<1, \\
x(a+) & =0
\end{aligned}
$$

has a solution given by

$$
x(t)=\mathscr{J}_{a+}^{q} g_{1}(t) .
$$

Lemma 5. Let $x \in C_{\delta}^{2}(J, \mathbb{R})$, and $g_{2} \in C(J, \mathbb{R})$. The fractional differential equation

$$
\begin{aligned}
\left({ }^{H} D_{a+}^{q}+\gamma^{H} D_{a+}^{q-1}\right) x(t) & =g_{2}(t), \quad t \in J, 1<q<2, \\
x(a+) & =x^{(1)}(a+)=0,
\end{aligned}
$$

has a solution given by

$$
x(t)=t^{-\gamma} \int_{a}^{t} s^{\gamma-1} g_{a+}^{q-1} g_{2}(s) d s
$$

Proof. Applying the fractional integral operator $\mathscr{J}_{a+}^{q}$ to (9), we have

$$
\begin{aligned}
\mathscr{J}_{a+}^{q} g_{2}(t)= & x(t)+b_{1}\left(\ln \frac{t}{a}\right)^{q-1}+b_{2}\left(\ln \frac{t}{a}\right)^{q-2} \\
& +\gamma \mathcal{F}_{a+}^{1}\left(x(t)+c_{1}\left(\ln \frac{t}{a}\right)^{q-2}\right),
\end{aligned}
$$

for some constants $b_{1}, b_{2}$, and $c_{1}$. The initial condition $x(a+)=$ 0 implies that $b_{2}=0$. Taking the first derivative of (11), it follows that

$$
\begin{aligned}
\frac{1}{t} \mathscr{J}_{a+}^{q-1} g_{2}(t)= & x^{(1)}(t)+b_{1}(q-1)\left(\ln \frac{t}{a}\right)^{q-2} \frac{1}{t} \\
& +\frac{\gamma}{t}\left(x(t)+c_{1}\left(\ln \frac{t}{a}\right)^{q-2}\right) .
\end{aligned}
$$

The condition $x^{(1)}(a+)=0$ implies that $b_{1}=0=c_{1}$. Multiplying (12) by the integrating factor given by

$$
\zeta(t)=e^{\int(\gamma / t) d t}=t^{\gamma},
$$

we get

$$
\frac{d}{d t}\left(t^{\gamma} x(t)\right)=t^{\gamma-1} \mathscr{J}_{a+}^{q-1} g_{2}(t)
$$

Integrating (14), and using again $x(a+)=0$, we conclude that

$$
x(t)=t^{-\gamma} \int_{a}^{t} s^{\gamma-1} \mathscr{J}_{a+}^{q-1} g_{2}(s) d s .
$$

This finishes the proof.

Remark 6. For $\gamma=0$, the solution is still valid, since $\mathscr{J}_{a+}^{q} g_{2}(t)=\mathscr{J}_{a+}^{1} \mathscr{J}_{a+}^{q-1} g_{2}(t)=\int_{a}^{t}\left(\mathscr{J}_{a+}^{q-1} g_{2}(s) / s\right) d s$. This trivial case will be negligible hereafter.

Lemma 7. Let $\lambda_{1}^{2} \geq 4 \lambda_{2}, x \in C_{\delta}^{3}(J, \mathbb{R})$, and $g_{3} \in C(J, \mathbb{R})$. The linear system

$$
\begin{aligned}
\left({ }^{H} D_{a+}^{q}+\lambda_{1}{ }^{H} D_{a+}^{q-1}+\lambda_{2}{ }^{H} D_{a+}^{q-2}\right) x(t) & =g_{3}(t), \\
t & \in J, 2<q<3,
\end{aligned}
$$

$$
x(a+)=x^{(1)}(a+)=x^{(2)}(a+)=0,
$$

has a solution

$$
\begin{aligned}
& x(t)=\frac{t^{-\lambda_{1} / 2}}{\sqrt{\lambda_{1}^{2}-4 \lambda_{2}}} \\
& \quad \int_{a}^{t}\left(\left(\frac{t}{s}\right)^{(1 / 2) \sqrt{\lambda_{1}^{2}-4 \lambda_{2}}}-\left(\frac{s}{t}\right)^{(1 / 2) \sqrt{\lambda_{1}^{2}-4 \lambda_{2}}}\right)
\end{aligned}
$$




$$
\begin{gathered}
\cdot s^{\lambda_{1} / 2+1} \mathcal{J}_{a+}^{q-2} g_{3}(s) d s, \quad \text { if } \lambda_{1}^{2}>4 \lambda_{2} \\
\text { or } x(t)=t^{-\lambda_{1} / 2} \int_{a}^{t} s^{\lambda_{1} / 2+1} \ln \frac{t}{s} \mathcal{J}_{a+}^{q-2} g_{3}(s) d s,
\end{gathered}
$$

$$
\text { if } \lambda_{1}^{2}=4 \lambda_{2} \text {. }
$$

Proof. Applying the fractional integral operator $\mathcal{J}_{a+}^{q}$ to (16), we have

$$
\begin{aligned}
x(t) & +b_{1}\left(\ln \frac{t}{a}\right)^{q-1}+b_{2}\left(\ln \frac{t}{a}\right)^{q-2}+b_{3}\left(\ln \frac{t}{a}\right)^{q-3} \\
& +\lambda_{1} \mathscr{J}_{a+}^{1}\left(x(t)+c_{2}\left(\ln \frac{t}{a}\right)^{q-2}+c_{3}\left(\ln \frac{t}{a}\right)^{q-3}\right) \\
& +\lambda_{2} \mathscr{J}_{a+}^{2}\left(x(t)+d_{3}\left(\ln \frac{t}{a}\right)^{q-3}\right)=\mathscr{J}_{a+}^{q} g_{3}(t),
\end{aligned}
$$

for some constants $b_{1}, b_{2}, b_{3}, c_{2}, c_{3}$, and $d_{3}$. The initial condition $x(a+)=0$ implies that $b_{3}=0$. Taking the first derivative of (18), and using Leibniz's rule, it follows that

$$
\begin{aligned}
x^{(1)}(t) & +b_{1}(q-1)\left(\ln \frac{t}{a}\right)^{q-2} \frac{1}{t} \\
& +b_{2}(q-2)\left(\ln \frac{t}{a}\right)^{q-3} \frac{1}{t} \\
& +\frac{\lambda_{1}}{t}\left(x(t)+c_{2}\left(\ln \frac{t}{a}\right)^{q-2}+c_{3}\left(\ln \frac{t}{a}\right)^{q-3}\right) \\
& +\frac{\lambda_{2}}{t} \int_{a}^{t}\left(x(s)+d_{3}\left(\ln \frac{s}{a}\right)^{q-3}\right) \frac{d s}{s} \\
= & \frac{1}{t} g_{a+}^{q-1} g_{3}(t) .
\end{aligned}
$$

The condition $x^{(1)}(a+)=0$ implies that $b_{2}=c_{3}=0$. Multiplying by $t$ and then the second derivative would imply

$$
\begin{gathered}
t x^{(2)}(t)+x^{(1)}(t)+b_{1}(q-1)(q-2)\left(\ln \frac{t}{a}\right)^{q-3} \frac{1}{t} \\
+\lambda_{1}\left(x^{(1)}(t)+c_{2}(q-2)\left(\ln \frac{t}{a}\right)^{q-3} \frac{1}{t}\right) \\
+\frac{\lambda_{2}}{t}\left(x(t)+d_{3}\left(\ln \frac{t}{a}\right)^{q-3}\right)=\frac{1}{t} \mathscr{J}_{a+}^{q-2} g_{3}(t) .
\end{gathered}
$$

For the last initial condition $x^{(2)}(a+)=0$, we get $b_{1}=c_{2}=$ $d_{3}=0$. Now, multiplying (20) by $t$, it follows that

$$
t^{2} x^{(2)}(t)+\left(\lambda_{1}+1\right) t x^{(1)}(t)+\lambda_{2} x(t)=\mathscr{J}_{a+}^{q-2} g_{3}(t),
$$

which is a second-order Cauchy-Euler differential equation that has a general solution $x(t)=x_{c}(t)+x_{p}(t)$, where $x_{c}(t)$ and $x_{p}(t)$ are the complementary and particular solution of (14), respectively. To find the complementary solution of (21), consider the solutions $x_{1}(t)=t^{m_{1}}$ and $x_{2}(t)=t^{m_{2}}$ for the homogeneous equation

$$
t^{2} x^{(2)}(t)+\left(\lambda_{1}+1\right) t x^{(1)}(t)+\lambda_{2} x(t)=0,
$$

where $m_{1}=\left(-\lambda_{1}-\sqrt{\lambda_{1}^{2}-4 \lambda_{2}}\right) / 2$ and $m_{2}=\left(-\lambda_{1}+\right.$ $\left.\sqrt{\lambda_{1}^{2}-4 \lambda_{2}}\right) / 2$ are the distinct real roots of the characteristic equation

$$
m^{2}+\lambda_{1} m+\lambda_{2}=0
$$

The complementary solution of the homogeneous equation (22) is

$$
x_{c}(t)=a_{1} t^{m_{1}}+a_{2} t^{m_{2}}
$$

for some constants $a_{1}$ and $a_{2}$. These two constants can be evaluated by the initial conditions $x(a+)=x^{(1)}(a+)=0$ given in (16), which both imply that

$$
\begin{aligned}
a_{1} a^{m_{1}}+a_{2} a^{m_{2}} & =0, \\
a_{1} m_{1} a^{m_{1}-1}+a_{2} m_{2} a^{m_{2}-1} & =0 .
\end{aligned}
$$

The only solution for these algebraic equations is $a_{1}=a_{2}=0$, since $m_{1} \neq m_{2}$. The Wronskian $W$ for the solutions $x_{1}$ and $x_{2}$ is

$$
\begin{aligned}
W\left(x_{1}, x_{2}\right) & =\left|\begin{array}{cc}
t^{m_{1}} & t^{m_{2}} \\
m_{1} t^{m_{1}-1} & m_{2} t^{m_{2}-1}
\end{array}\right| \\
& =\left(m_{2}-m_{1}\right) t^{m_{1}+m_{2}-1}=\sqrt{\lambda_{1}^{2}-4 \lambda_{2}} t^{-\lambda_{1}-1} \\
& \neq 0,
\end{aligned}
$$

since $\lambda_{1}^{2}>4 \lambda_{2}$. Applying the variation of parameter technique we can get the particular solution

$$
x_{p}(t)=\int_{a}^{t} \frac{t^{m_{2}} s^{m_{1}}-t^{m_{1}} s^{m_{2}}}{\left(m_{2}-m_{1}\right) s^{m_{1}+m_{2}-1}} \mathscr{J}_{a+}^{q-2} g_{3}(s) d s .
$$

Therefore, the general solution is $x(t)=x_{p}(t)$.

If $\lambda_{1}^{2}=4 \lambda_{2}$, we consider $x_{1}(t)=t^{-\lambda_{1} / 2}$ and $x_{2}(t)=$ $t^{-\lambda_{1} / 2} \ln t$. Then, the complementary and the particular solutions are

$$
\begin{aligned}
& x_{c}(t)=a_{1} t^{-\lambda_{1} / 2}+a_{2} t^{-\lambda_{1} / 2} \ln t, \\
& x_{p}(t)=t^{-\lambda_{1} / 2} \int_{a}^{t} s^{\lambda_{1} / 2+1} \ln \frac{t}{s} \mathcal{J}_{a+}^{q-2} g_{3}(s) d s,
\end{aligned}
$$

where the Wronskian in this case is given by

$$
\begin{aligned}
W & \left(x_{1}, x_{2}\right) \\
& =\left|\begin{array}{cc}
t^{-\lambda_{1} / 2} & t^{-\lambda_{1} / 2} \ln t \\
-\frac{\lambda_{1}}{2} t^{-\lambda_{1} / 2-1} & -\frac{\lambda_{1}}{2} t^{-\lambda_{1} / 2-1} \ln t+t^{-\lambda_{1} / 2-1}
\end{array}\right| \\
& =-\frac{\lambda_{1}}{2} t^{-\lambda_{1}-1} \ln t+t^{-\lambda_{1}-1}+\frac{\lambda_{1}}{2} t^{-\lambda_{1}-1} \ln t=t^{-\lambda_{1}-1} \\
& \neq 0 .
\end{aligned}
$$


The evaluation of the constants $a_{1}$ and $a_{2}$ in the complementary solution (28) leads to

$$
\begin{aligned}
a_{1} a^{-\lambda_{1} / 2}+a_{2} a^{-\lambda_{1} / 2} \ln a & =0, \\
-\frac{\lambda_{1}}{2} a_{1} a^{-\lambda_{1} / 2-1}-\frac{\lambda_{1}}{2} a_{2} a^{-\lambda_{1} / 2-1} \ln a+a_{2} a^{-\lambda_{1} / 2-1} & =0,
\end{aligned}
$$

which imply $a_{1}=a_{2}=0$. Therefore, the general solution becomes again $x(t)=x_{p}(t)$. The two cases together constitute the required solution. This finishes the proof.

Remark 8. If $\lambda_{1}^{2}<4 \lambda_{2}$, then (23) has two complex conjugate roots that will not be considered in this article. On the other hand, if $\lambda_{1}=\lambda_{2}=0$, the solution is $x(t)=\mathscr{J}_{a+}^{q} g_{3}(t)$, which is a trivial case and hereafter will be negligible.

Remark 9. Any of initial conditions $x(a+)=x^{(1)}(a+)=$ $x^{(2)}(a+)=$ constant $\neq 0$ should not be used because it would imply ill-posed systems.

\section{Existence Theorems}

We establish sufficient conditions for existence of solutions to problems (1)-(3) using different types of fixed-point theorems.

In view of Lemmas 4, 5, and 7, we transform the initial value problems (1)-(3), respectively, into operator equations as

$$
\begin{aligned}
& \Psi_{1} x(t)=\mathscr{J}_{a+}^{q} f_{1}(t, x(t)), \quad 0<q<1, \\
& \Psi_{2} x(t)=t^{-\gamma} \int_{a}^{t} s^{\gamma-1} \mathscr{J}_{a+}^{q-1} f_{2}(s, x(s)) d s, \quad 1<q<2,
\end{aligned}
$$

and for $2<q<3$, we define the respective cases $\lambda_{1}^{2}>4 \lambda_{2}$, and $\lambda_{1}^{2}=4 \lambda_{2}$, as

$$
\begin{aligned}
& \Psi_{3} x(t)=\frac{t^{-\lambda_{1} / 2}}{\sqrt{\lambda_{1}^{2}-4 \lambda_{2}}} \\
& \cdot \int_{a}^{t}\left(\left(\frac{t}{s}\right)^{(1 / 2) \sqrt{\lambda_{1}^{2}-4 \lambda_{2}}}-\left(\frac{s}{t}\right)^{(1 / 2) \sqrt{\lambda_{1}^{2}-4 \lambda_{2}}}\right) \\
& \cdot s^{\lambda_{1} / 2+1} \mathscr{g}_{a+}^{q-2} g_{3}(s) d s, \quad \text { if } \lambda_{1}^{2}>4 \lambda_{2} \\
& \text { or } \Psi_{3} x(t)=t^{-\lambda_{1} / 2} \int_{a}^{t} s^{\lambda_{1} / 2+1} \ln \frac{t}{s} \mathcal{g}_{a+}^{q-2} g_{3}(s) d s, \\
& \text { if } \lambda_{1}^{2}=4 \lambda_{2} .
\end{aligned}
$$

where $t \in J$. If the operator $\Psi_{k}, k=1,2,3$, has a fixed point, the corresponding problem in systems (1)-(3) has this fixed point as a solution.

Lemma 10. The operator $\Psi_{k}: C(J, \mathbb{R}) \rightarrow C(J, \mathbb{R}), k=1,2,3$, is completely continuous.

Proof. Obviously, the continuity of the operator $\Psi_{k}$ follows from the continuity of the function $f_{k}$. Let $\mathcal{U}$ be a bounded proper subset of $C(J, \mathbb{R})$; then for any $t \in J$, and $x \in \mathcal{U}$, there exists a positive constant $L_{k}$ such that $\left|f_{k}(t, x(t))\right| \leq L_{k}$. Accordingly, for any $t \in J$, and $x \in \mathcal{U}$, it follows that

$$
\begin{aligned}
& \left|\Psi_{1} x(t)\right| \leq \frac{L_{1}(\ln (t / a))^{q}}{\Gamma(q+1)}, \quad 0<q<1, \\
& \left|\Psi_{2} x(t)\right| \leq \frac{L_{2}(\ln (t / a))^{q-1}\left|1-(a / t)^{\gamma}\right|}{|\gamma| \Gamma(q)}, \\
& \quad 1<q<2, \gamma \neq 0,
\end{aligned}
$$

and for $2<q<3$, we have

$$
\begin{aligned}
& \left|\Psi_{3} x(t)\right| \leq \frac{L_{3} a^{2}(\ln (t / a))^{q-2}}{\sqrt{\lambda_{1}^{2}-4 \lambda_{2}} \Gamma(q-1)} \\
& \times \mid \frac{(t / a)^{2}-(t / a)^{-\lambda_{1} / 2+(1 / 2) \sqrt{\lambda_{1}^{2}-4 \lambda_{2}}}}{2+\lambda_{1} / 2-(1 / 2) \sqrt{\lambda_{1}^{2}-4 \lambda_{2}}} \\
& -\frac{(t / a)^{2}-(a / t)^{\lambda_{1} / 2+(1 / 2) \sqrt{\lambda_{1}^{2}-4 \lambda_{2}}}}{2+\lambda_{1} / 2+(1 / 2) \sqrt{\lambda_{1}^{2}-4 \lambda_{2}}} \mid, \quad \text { if } \lambda_{1}^{2}>4 \lambda_{2}, \\
& \left|\Psi_{3} x(t)\right| \leq \frac{L_{3} a^{2}(\ln (t / a))^{q-1}}{\Gamma(q-1)}\left|\frac{(t / a)^{2}-(a / t)^{\lambda_{1} / 2}}{\lambda_{1} / 2+2}\right|,
\end{aligned}
$$

Consequently, for any $k=1,2,3$, the operator $\Psi_{k}$ is bounded on $\mathcal{U} \subset C(J, \mathbb{R})$. Furthermore, if $t_{1}, t_{2} \in J$, such that $a \leq t_{1}<$ $t_{2} \leq b$, then

$$
\begin{aligned}
& \left|\left(\Psi_{1} x\right)\left(t_{2}\right)-\left(\Psi_{1} x\right)\left(t_{1}\right)\right| \\
& \quad \leq \frac{L_{1}\left|\left(\ln \left(t_{2} / a\right)\right)^{q}-\left(\ln \left(t_{1} / a\right)\right)^{q}\right|}{\Gamma(q+1)}
\end{aligned}
$$

for $0<q<1$. Let $1<q<2$, and then

$$
\begin{aligned}
& \left|\left(\Psi_{2} x\right)\left(t_{2}\right)-\left(\Psi_{2} x\right)\left(t_{1}\right)\right| \leq \frac{L_{2}}{|\gamma| \Gamma(q)} \\
& \quad \times\left(\left(t_{1}^{\gamma}-a^{\gamma}\right)\left(\ln \left(\frac{t_{1}}{a}\right)\right)^{q-1}+t_{2}^{\gamma}\left(\ln \left(\frac{t_{2}}{a}\right)\right)^{q-1}\right) \\
& \cdot\left|t_{2}^{\gamma}-t_{1}^{\gamma}\right|, \quad \gamma \neq 0 .
\end{aligned}
$$

For $2<q<3$, let $\lambda_{1}^{2}>4 \lambda_{2}$, and then

$$
\begin{aligned}
& \left|\left(\Psi_{3} x\right)\left(t_{2}\right)-\left(\Psi_{3} x\right)\left(t_{1}\right)\right| \\
& \quad \leq \frac{\left|t_{2}^{-\lambda_{1} / 2+(1 / 2) \sqrt{\lambda_{1}^{2}-4 \lambda_{2}}}-t_{1}^{-\lambda_{1} / 2+(1 / 2) \sqrt{\lambda_{1}^{2}-4 \lambda_{2}}}\right|}{\sqrt{\lambda_{1}^{2}-4 \lambda_{2}}} \\
& \quad \times \int_{a}^{t_{1}} s^{\lambda_{1} / 2+1-(1 / 2) \sqrt{\lambda_{1}^{2}-4 \lambda_{2}}}\left|\mathscr{F}_{a+}^{q-2} f_{3}(s, x(s))\right| d s
\end{aligned}
$$




$$
\begin{aligned}
& +\frac{\left|t_{2}^{-\lambda_{1} / 2-(1 / 2)} \sqrt{\lambda_{1}^{2}-4 \lambda_{2}}-t_{1}^{-\lambda_{1} / 2-(1 / 2) \sqrt{\lambda_{1}^{2}-4 \lambda_{2}}}\right|}{\sqrt{\lambda_{1}^{2}-4 \lambda_{2}}} \\
& \times \int_{a}^{t_{1}} s^{\lambda_{1} / 2+1+(1 / 2) \sqrt{\lambda_{1}^{2}-4 \lambda_{2}}}\left|\mathcal{F}_{a+}^{q-2} f_{3}(s, x(s))\right| d s \\
& +\frac{t_{2}^{-\lambda_{1} / 2+(1 / 2) \sqrt{\lambda_{1}^{2}-4 \lambda_{2}}}}{\sqrt{\lambda_{1}^{2}-4 \lambda_{2}}} \\
& \times \int_{t_{1}}^{t_{2}} s^{\lambda_{1} / 2+1-(1 / 2) \sqrt{\lambda_{1}^{2}-4 \lambda_{2}}}\left|\mathscr{F}_{a+}^{q-2} f_{3}(s, x(s))\right| d s \\
& +\frac{t_{2}^{-\lambda_{1} / 2-(1 / 2) \sqrt{\lambda_{1}^{2}-4 \lambda_{2}}}}{\sqrt{\lambda_{1}^{2}-4 \lambda_{2}}} \\
& \times \int_{t_{1}}^{t_{2}} s^{\lambda_{1} / 2+1+(1 / 2) \sqrt{\lambda_{1}^{2}-4 \lambda_{2}}}\left|\mathscr{J}_{a+}^{q-2} f_{3}(s, x(s))\right| d s \\
& \leq \frac{L_{3}\left|t_{2}^{-\lambda_{1} / 2+(1 / 2) \sqrt{\lambda_{1}^{2}-4 \lambda_{2}}}-t_{1}^{-\lambda_{1} / 2+(1 / 2) \sqrt{\lambda_{1}^{2}-4 \lambda_{2}}}\right|\left(\ln \left(t_{1} / a\right)\right)^{q-2}}{\sqrt{\lambda_{1}^{2}-4 \lambda_{2}} \Gamma(q-1)}
\end{aligned}
$$

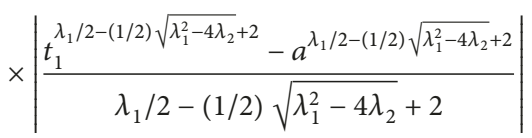

$$
\begin{aligned}
& +\frac{L_{3}\left|t_{2}^{-\lambda_{1} / 2-(1 / 2) \sqrt{\lambda_{1}^{2}-4 \lambda_{2}}}-t_{1}^{-\lambda_{1} / 2-(1 / 2) \sqrt{\lambda_{1}^{2}-4 \lambda_{2}}}\right|\left(\ln \left(t_{1} / a\right)\right)^{q-2}}{\sqrt{\lambda_{1}^{2}-4 \lambda_{2}} \Gamma(q-1)}
\end{aligned}
$$

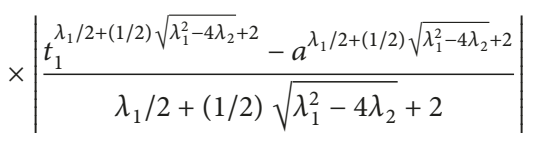

$$
\begin{aligned}
& +\frac{L_{3} t_{2}^{-\lambda_{1} / 2+(1 / 2) \sqrt{\lambda_{1}^{2}-4 \lambda_{2}}}\left(\ln \left(t_{2} / a\right)\right)^{q-2}}{\sqrt{\lambda_{1}^{2}-4 \lambda_{2}} \Gamma(q-1)} \\
& \times\left|\frac{t_{2}^{\lambda_{1} / 2-(1 / 2) \sqrt{\lambda_{1}^{2}-4 \lambda_{2}}+2}-t_{1}^{\lambda_{1} / 2-(1 / 2) \sqrt{\lambda_{1}^{2}-4 \lambda_{2}}+2}}{\lambda_{1} / 2-(1 / 2) \sqrt{\lambda_{1}^{2}-4 \lambda_{2}}+2}\right| \\
& +\frac{L_{3} t_{2}^{-\lambda_{1} / 2-(1 / 2) \sqrt{\lambda_{1}^{2}-4 \lambda_{2}}}\left(\ln \left(t_{2} / a\right)\right)^{q-2}}{\sqrt{\lambda_{1}^{2}-4 \lambda_{2}} \Gamma(q-1)} \\
& \times\left|\frac{t_{2}^{\lambda_{1} / 2+(1 / 2) \sqrt{\lambda_{1}^{2}-4 \lambda_{2}}+2}-t_{1}^{\lambda_{1} / 2+(1 / 2) \sqrt{\lambda_{1}^{2}-4 \lambda_{2}}+2}}{\lambda_{1} / 2+(1 / 2) \sqrt{\lambda_{1}^{2}-4 \lambda_{2}}+2}\right| .
\end{aligned}
$$

Now, let $\lambda_{1}^{2}=4 \lambda_{2}$, and then

$$
\begin{gathered}
\left|\left(\Psi_{3} x\right)\left(t_{2}\right)-\left(\Psi_{3} x\right)\left(t_{1}\right)\right| \leq\left|t_{2}^{-\lambda_{1} / 2} \ln \frac{t_{2}}{a}-t_{1}^{-\lambda_{1} / 2} \ln \frac{t_{1}}{a}\right| \\
\cdot \int_{a}^{t_{1}} s^{\lambda_{1} / 2+1}\left|\mathscr{g}_{a+}^{q-2} f_{3}(s, x(s))\right| d s+t_{2}^{-\lambda_{1} / 2} \ln \frac{t_{2}}{a}
\end{gathered}
$$

$$
\begin{aligned}
& \cdot \int_{t_{1}}^{t_{2}} s^{\lambda_{1} / 2+1}\left|\mathscr{J}_{a+}^{q-2} f_{3}(s, x(s))\right| d s \\
& \leq L_{3}\left|t_{2}^{-\lambda_{1} / 2} \ln \frac{t_{2}}{a}-t_{1}^{-\lambda_{1} / 2} \ln \frac{t_{1}}{a}\right| \frac{\left|t_{1}^{\lambda_{1} / 2+2}-a^{\lambda_{1} / 2+2}\right|}{\lambda_{1} / 2+2} \\
& \cdot \frac{\left(\ln \left(t_{1} / a\right)\right)^{q-2}}{\Gamma(q-1)}+L_{3} t_{2}^{-\lambda_{1} / 2} \\
& \cdot \ln \frac{t_{2}}{a} \frac{\left|t_{2}^{\lambda_{1} / 2+2}-t_{1}^{\lambda_{1} / 2+2}\right|}{\lambda_{1} / 2+2} \frac{\left(\ln \left(t_{2} / a\right)\right)^{q-2}}{\Gamma(q-1)} .
\end{aligned}
$$

As $t_{2} \rightarrow t_{1},\left|\left(\Psi_{k} x\right)\left(t_{2}\right)-\left(\Psi_{k} x\right)\left(t_{1}\right)\right| \rightarrow 0$, for $k=1,2,3$. This implies that $\Psi_{k}$ is equicontinuous on $J$. In consequence, it follows by the Arzela-Ascoli theorem that the operator $\Psi_{k}$ is completely continuous. This finishes the proof. ([14]).

We need the following Schauder's fixed point theorem

Theorem 11. If $\mathcal{U}$ is a closed, bounded, convex subset of a Banach space $\mathscr{X}$ and the mapping $\Delta: \mathcal{U} \rightarrow \mathcal{U}$ is completely continuous, then $\Delta$ has a fixed point in $\mathcal{U}$.

Accordingly, if we define a closed, bounded, convex subset $\mathcal{U}$ of $C(J, \mathbb{R})$ on which $\Psi_{k}^{\prime}$ s, as defined by (31)-(32), are completely continuous, then problems (1)-(3) have the respective solution.

Theorem 12. Assume that

$$
\lim _{x \rightarrow 0} \frac{f_{k}(t, x)}{x} \leq \xi_{k}<\infty, \quad t \in J
$$

then, each problem of (1)-(3) has a solution.

Proof. For any $k$, the given condition implies that $\mid f_{k}(t$, $x(t))\left|\leq\left(1+\xi_{k}\right)\right| x(t) \mid$, whenever $|x(t)|<\delta_{k}$, for a fixed number $\delta_{k}>0$. Therefore, define a subset $\mathscr{U}_{k}$ as

$$
\mathscr{U}_{k}=\left\{x \in C(J, \mathbb{R}):|x(t)| \leq \delta_{k}, t \in J\right\} .
$$

Hence, $\mathscr{U}_{k}$ is a closed, bounded, and convex subset of $C(J, \mathbb{R})$. If $x \in \mathscr{U}_{k}$, then $\left|f_{k}(t, x(t))\right|<\delta_{k}\left(1+\xi_{k}\right)$, for any $t \in J$. On the other hand, the operator $\Psi_{k}: \mathscr{U}_{k} \rightarrow \mathscr{U}_{k}$ is completely continuous by Lemma 10; then by Schauder's fixed-point Theorem 11, each problem of (1)-(3) has a solution. This finishes the proof.

Our next result deals with the uniqueness of solution for problems (1)-(3) and is based on the Banach contraction theorem. The following assumptions are essential for proving the next theorem.

(H1) For any $t \in J, x, y \in \mathbb{R}, k=1,2,3$,

$$
\begin{aligned}
&\left|f_{k}(t, x)-f_{k}(t, y)\right| \leq B_{k}|x-y|, \\
& \sup _{t \in J}\left|f_{k}(t, 0)\right|=A_{k}, \\
& C_{k}=\max \left\{A_{k}, B_{k}\right\} .
\end{aligned}
$$


(H2) Let $\eta_{k}<1$, such that

$$
\begin{aligned}
& \eta_{1}=\frac{C_{1}(\ln (b / a))^{q}}{\Gamma(q+1)}, \\
& \eta_{2}=\frac{C_{2}(\ln (b / a))^{q-1} \max _{t \in J}\left|1-(a / t)^{\gamma}\right|}{|\gamma| \Gamma(q)}, \quad \gamma \neq 0, \\
& \eta_{3}=\frac{C_{3}(\ln (b / a))^{q-2} L}{\Gamma(q-1)} .
\end{aligned}
$$

Here $L=M$, if $\lambda_{1}^{2}>4 \lambda_{2}$, or $L=N$, if $\lambda_{1}^{2} \neq 4 \lambda_{2}$, where

$$
\begin{aligned}
N & =\max _{t \in J}\left|\frac{t^{2}-a^{2}(a / t)^{\lambda_{1} / 2}}{\lambda_{1} / 2+2}\right| \ln \left(\frac{t}{a}\right), \\
M & =\frac{1}{\sqrt{\lambda_{1}^{2}-4 \lambda_{2}}} \\
& \cdot \max _{t \in J} \mid \frac{t^{2}-\left(1 / a^{2}\right)(t / a)^{-\lambda_{1} / 2+(1 / 2) \sqrt{\lambda_{1}^{2}-4 \lambda_{2}}}}{2+\lambda_{1} / 2-(1 / 2) \sqrt{\lambda_{1}^{2}-4 \lambda_{2}}} \\
& -\frac{t^{2}-\left(1 / a^{2}\right)(a / t)^{\lambda_{1} / 2+(1 / 2) \sqrt{\lambda_{1}^{2}-4 \lambda_{2}}}}{2+\lambda_{1} / 2+(1 / 2) \sqrt{\lambda_{1}^{2}-4 \lambda_{2}}} \mid .
\end{aligned}
$$

Theorem 13. Assume that (H1) and (H2) are satisfied. Then there exists a unique solution for each of the problems (1)-(3) on $J$.

Proof. Let $r_{k} \geq\left(1-\eta_{k}\right)^{-1} \eta_{k}$, for $k=1,2,3$. We show that $\Psi_{k} \mathscr{B}_{r_{k}} \subset \mathscr{B}_{r_{k}}$, where $\mathscr{B}_{r_{k}}=\left\{x \in C(J, \mathbb{R}):\|x\| \leq r_{k}\right\}$. This follows by the following estimates:

$$
\begin{aligned}
& \left|\Psi_{1} x(t)\right| \leq \frac{B_{1}(\ln (b / a))^{q}}{\Gamma(q+1)}\|x\|+\frac{A_{1}(\ln (b / a))^{q}}{\Gamma(q+1)} \\
& \leq\left(1-\eta_{1}\right) r_{1}+\eta_{1} r_{1}=r_{1}, \\
& \left|\Psi_{2} x(t)\right| \\
& \leq \frac{B_{2}(\ln (b / a))^{q-1}\left|1-(a / t)^{\gamma}\right|}{|\gamma| \Gamma(q)}\|x\| \\
& \quad+\frac{A_{2}(\ln (b / a))^{q-1}\left|1-(a / t)^{\gamma}\right|}{|\gamma| \Gamma(q)} \leq r_{2},
\end{aligned}
$$

$\left|\Psi_{3} x(t)\right|$

$\leq \begin{cases}\frac{C_{3}(\ln (b / a))^{q-2} M}{\Gamma(q-1)}\left(A_{3}+B_{3}\|x\|\right), & \text { if } \lambda_{1}^{2}>4 \lambda_{2}, \\ \frac{C_{3}(\ln (t / a))^{q-2} N}{\Gamma(q-1)}\left(A_{3}+B_{3}\|x\|\right), & \text { if } \lambda_{1}^{2}=4 \lambda_{2}\end{cases}$

$\leq r_{3}$ for any $x \in \mathscr{B}_{r_{k}}$. Moreover, for $x, y \in C(J, \mathbb{R}), k=1,2,3$, and for each $t \in J$, we obtain

$$
\left|\left(\Psi_{k} x\right)(t)-\left(\Psi_{k} y\right)(t)\right| \leq \eta_{k}\|x-y\| .
$$

As $\eta_{k}<1, \Psi_{k}$ is a contraction. Thus, the conclusion of the theorem follows by the contraction mapping principle. This completes the proof.

We close the existence theorems by applying LeraySchauder degree theorem [14]. Therefore, we need the following assumption.

(H3) For $t \in J, x \in \mathbb{R}$, and $k=1,2,3$,

$$
\left|f_{k}(t, x)\right| \leq D_{k}(|x|+1)
$$

where $D_{k}<\infty$, satisfies

$$
\begin{aligned}
& F_{1}=\frac{D_{1}(\ln (b / a))^{q}}{\Gamma(q+1)}<1, \\
& F_{2}=\frac{D_{2}(\ln (b / a))^{q-1} \max _{t \in J}\left|1-(a / t)^{\gamma}\right|}{|\gamma| \Gamma(q)}<1, \\
& F_{3}=\frac{D_{3}(\ln (b / a))^{q-2} L}{\Gamma(q-1)}<1, \quad \gamma \neq 0,
\end{aligned}
$$

where $L$ is defined as in (H2).

Theorem 14. Assume that (H3) is satisfied. Then every problem of (1)-(3) has a solution.

Proof. Define a ball $\mathscr{B}_{R_{k}}=\left\{x \in C(J, \mathbb{R}):\|x\|<R_{k}\right\}$, $k=1,2,3$, for some positive real number $R_{k}$ which will be determined later. We show that $\Psi_{k}: \overline{\mathscr{B}_{R_{k}}} \rightarrow C(J, \mathbb{R})$ satisfies

$$
0 \notin\left(I-\lambda_{k} \Psi_{k}\right)\left(\partial \mathscr{B}_{R_{k}}\right),
$$

for any $x \in \partial \mathscr{B}_{R_{k}}$, and $\lambda_{k} \in[0,1]$; here $\partial \mathscr{B}_{R_{k}}$ denotes the boundary set of $\mathscr{B}_{R_{k}}$. Define the homotopy

$$
\begin{aligned}
& h_{\lambda_{k}}(x)=H\left(\lambda_{k}, x\right)=x-\lambda_{k} \Psi_{k} x, \\
& x \in C(J, \mathbb{R}), \lambda_{k} \in[0,1] .
\end{aligned}
$$

Then, by Lemma $10, h_{\lambda_{k}}$ is completely continuous. Let $I$ denote the identity operator; then the homotopy invariance and normalization properties of topological degrees imply that

$$
\begin{aligned}
\operatorname{deg}\left(h_{\lambda_{k}}, \mathscr{B}_{R_{k}}, 0\right) & =\operatorname{deg}\left(\left(I-\lambda_{k} \Psi_{k}\right), \mathscr{B}_{R_{k}}, 0\right) \\
& =\operatorname{deg}\left(h_{1}, \mathscr{B}_{R_{k}}, 0\right) \\
& =\operatorname{deg}\left(h_{0}, \mathscr{B}_{R_{k}}, 0\right) \\
& =\operatorname{deg}\left(I, \mathscr{B}_{R_{k}}, 0\right)=1
\end{aligned}
$$


since $0 \in \mathscr{B}_{R_{k}}$. By the nonzero property of the LeraySchauder degree, $h_{1}(x)=x-\Psi_{k} x=0$ for at least one $x \in \mathscr{B}_{R_{k}}$. To find $R_{k}$, we assume that $x(t)=\lambda_{k} \Psi_{k} x(t)$ for some $\lambda_{k} \in[0,1]$ and for all $t \in J$. Then, using (H3), we have

$$
|x(t)|=\left|\lambda_{k} \Psi_{k} x(t)\right| \leq F_{k}(1+\|x\|) .
$$

Therefore,

$$
\|x\| \leq \frac{F_{k}}{1-F_{k}} .
$$

The value of $R_{k}=1 /\left(1-F_{k}\right)>\|x\|$ is sufficient for applicability of Leray-Schauder degree theorem. This completes the proof.

Example 1. Consider the following nonlinear fractional differential equation:

$$
\begin{aligned}
\left({ }^{H} D_{2+}^{1.2}-4^{H} D_{2+}^{0.2}\right) x(t) & =t \sin x(t), \quad t \in(2,3], \\
x(2) & =x^{\prime}(2)=0 .
\end{aligned}
$$

Here $q=1.2, f_{2}(t, x(t))=(t / 3) \sin x(t)$, and $\gamma=-4$. Simple calculations lead to $\xi_{2}=C_{2}=D_{2}=1$, and

$$
\begin{aligned}
\eta_{2} & =\frac{C_{2}(\ln (b / a))^{q-1}\left|1-(a / t)^{\gamma}\right|}{|\gamma| \Gamma(q)} \\
& =\frac{(\ln (3 / 2))^{0.2} \max _{t \in J}\left|1-(2 / t)^{-4}\right|}{4(0.9)}=0.95 .
\end{aligned}
$$

Therefore, all previous existence theorems ensure the existence and uniqueness of the solution in $C([2,3], \mathbb{R})$ for problem (53).

Example 2. Consider the following nonlinear fractional differential equation:

$$
\begin{aligned}
& \left({ }^{H} D_{1+}^{2.6}-2^{H} D_{1+}^{1.6}-{ }^{H} D_{1+}^{0.6}\right) x(t)=\frac{e^{-3.2}|x(t)|}{1+|x(t)|}, \\
& M=\frac{1}{2 \sqrt{2}} \max _{t \in J}\left|\frac{t^{2}-t^{1+\sqrt{2}}}{1-\sqrt{2}}-\frac{t^{2}-t^{1-\sqrt{2}}}{1+\sqrt{2}}\right| \leq 20.4, \\
& \eta_{3}=\frac{C_{3}(\ln (b / a))^{q-2} L}{\Gamma(q-1)}=\frac{20.4 e^{-3.2}}{\Gamma(1.6)}=0.94 .
\end{aligned}
$$$$
t \in(1, e] \text {, }
$$

We apply any of the following:

(1) Theorem 12, with $\xi_{3}=e^{-3.2}$

(2) Theorem 13, with $C_{3}=e^{-3.2}$, and $\eta_{3}<1$

(3) Theorem 14, with $D_{3} \leq e^{-3.2}$, and $F_{3}=0.94<1$

Problem $(55)$ has a solution in $C([1, e], \mathbb{R})$. The uniqueness of this solution follows from Theorem 13.

\section{Conflicts of Interest}

The author declares that there are no conflicts of interest regarding the publication of this paper.

\section{References}

[1] F. C. Meral, T. J. Royston, and R. Magin, "Fractional calculus in viscoelasticity: an experimental study," Communications in Nonlinear Science and Numerical Simulation, vol. 15, no. 4, pp. 939-945, 2010.

[2] K. B. Oldham, "Fractional differential equations in electrochemistry," Advances in Engineering Software, vol. 41, no. 1, pp. 9-12, 2010.

[3] K. Balachandran, M. Matar, and J. J. Trujillo, "Note on controllability of linear fractional dynamical systems," Journal of Control and Decision, vol. 3, no. 4, pp. 267-279, 2016.

[4] B. Ahmad, M. M. Matar, and R. P. Agarwal, "Existence results for fractional differential equations of arbitrary order with nonlocal integral boundary conditions," Boundary Value Problems, vol. 2015, no. 1, article 220, pp. 1-13, 2015.

[5] B. Ahmad and J. J. Nieto, "Riemann-Liouville fractional integrodifferential equations with fractional nonlocal integral boundary conditions," Boundary Value Problems, vol. 2011, no. 36, 2011.

[6] B. Ahmad and S. K. Ntouyas, "On Hadamard fractional integrodifferential boundary value problems," Applied Mathematics and Computation, vol. 47, no. 1-2, pp. 119-131, 2015.

[7] A. Alsaedi, S. K. Ntouyas, B. Ahmad, and A. Hobiny, "Nonlinear Hadamard fractional differential equations with Hadamard type nonlocal non-conserved conditions," Advances in Difference Equations, vol. 2015, no. 1, article 285, 2015.

[8] B. Ahmad, M. M. Matar, and S. K. Ntouyas, "On general fractional differential inclusions with nonlocal integral boundary conditions," Differential Equations and Dynamical Systems, pp. $1-14,2016$.

[9] M. Matar, "On existence of positive solution for initial value problem of nonlinear fractional differential equations of order $1<\alpha \leq 2$," Acta Mathematica Universitatis Comenianae, vol. LXXXIV, no. 1, pp. 51-57, 2015.

[10] A. Alsaedi, S. K. Ntouyas, R. P. Agarwal, and B. Ahmad, "On Caputo type sequential fractional differential equations with nonlocal integral boundary conditions," Advances in Difference Equations, vol. 2015, no. 1, pp. 1-12, 2015.

[11] J. Hadamard, "Essai sur letude des fonctions donnees par leur developpment de Taylor," Journal de Mathématiques Pures et Appliquées, vol. 8, pp. 101-186, 1892.

[12] A. A. Kilbas, H. M. Srivastava, and J. J. Trujillo, Theory and Applications of Fractional Differential Equations, New York, NY, USA, Elsevier, 2006.

[13] A. A. Kilbas, "Hadamard-type fractional calculus," Journal of the Korean Mathematical Society, vol. 38, no. 6, pp. 1191-1204, 2001.

[14] D. R. Smart, Fixed Point Theorems, Cambridge University Press, London, UK, 1980. 


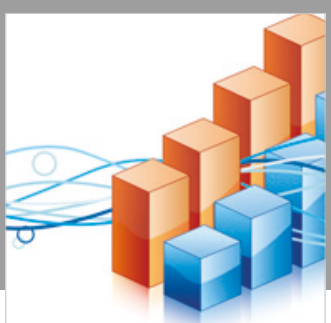

Advances in

Operations Research

\section{-n-m}
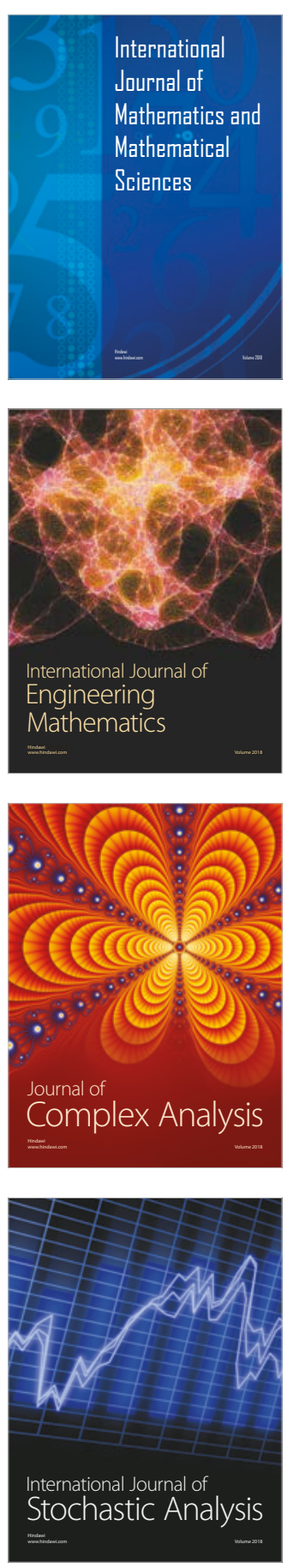
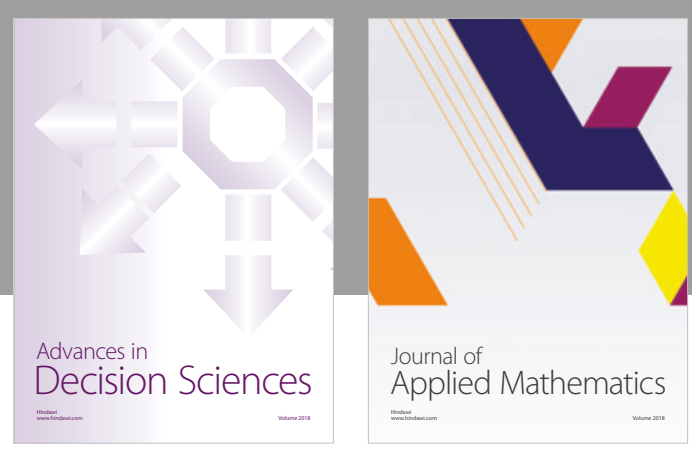

Journal of

Applied Mathematics
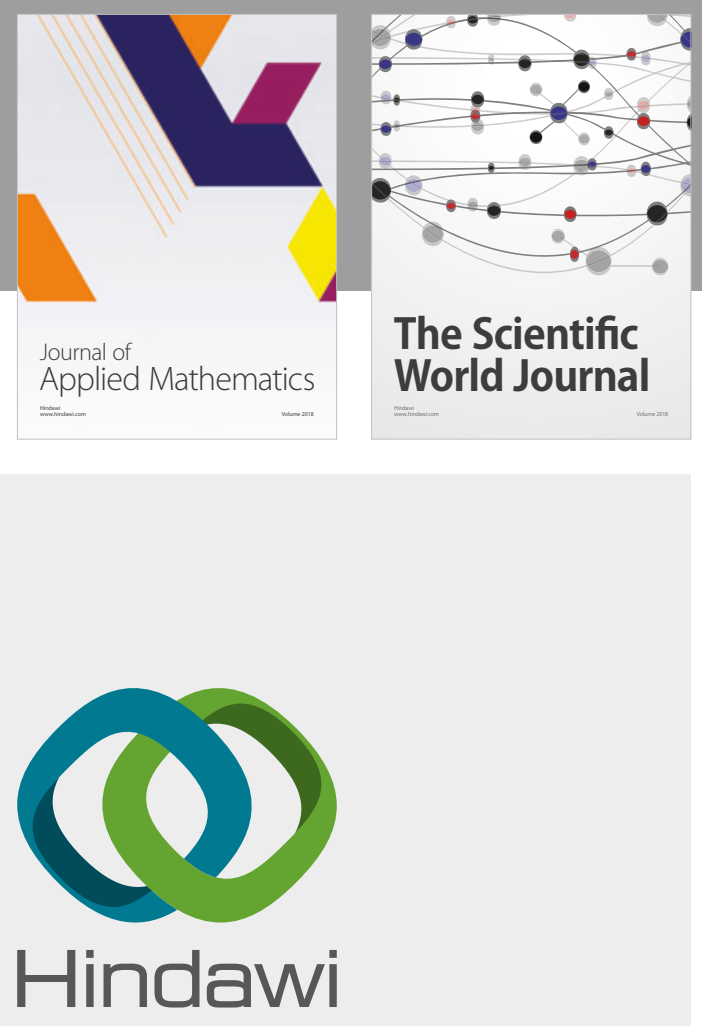

Submit your manuscripts at

www.hindawi.com

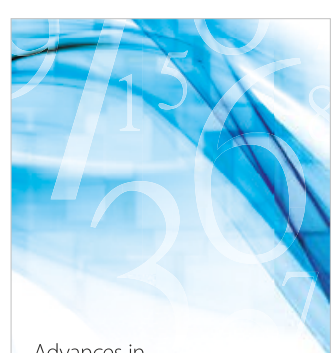

Advances in
Numerical Analysis
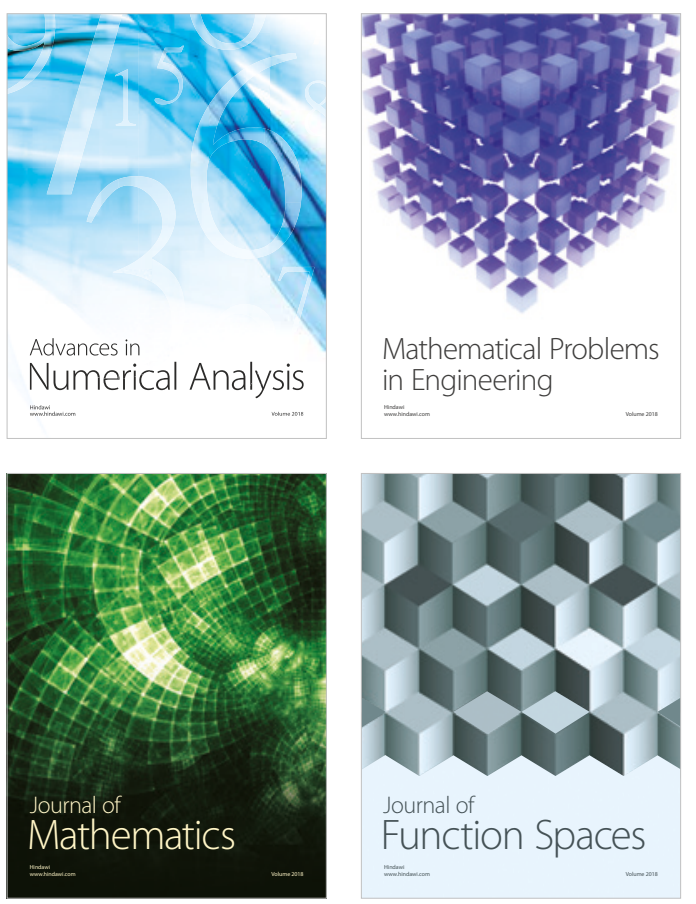

Mathematical Problems in Engineering

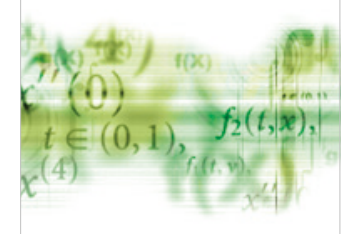

International Journal of

Differential Equations

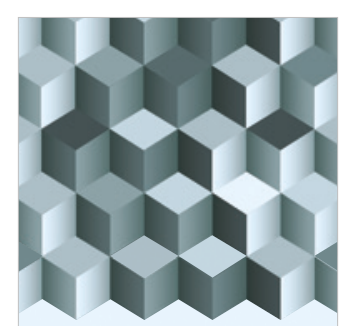

Journal of

Function Spaces

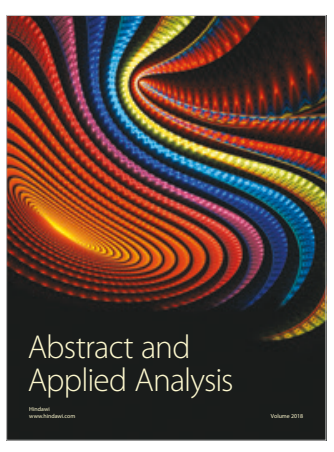

The Scientific

World Journal

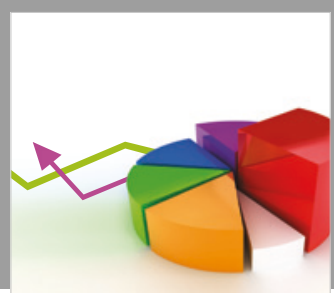

Journal of

Probability and Statistics
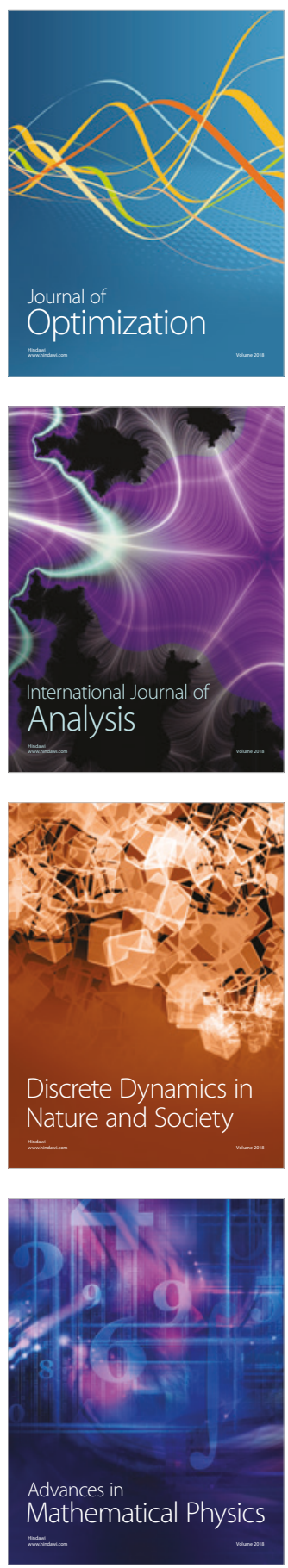